\section{Polyhedral Regions of Local Stability for Linear Discrete-Time Systems with Saturating Controls}

João Manoel Gomes da Silva, Jr., and Sophie Tarbouriech

\begin{abstract}
The study and the determination of polyhedral regions of local stability for linear systems subject to control saturation is addressed. The analysis of the nonlinear behavior of the closed-loop saturated system is made by dividing the state space in regions of saturation. Inside each of these regions, the system evolution can be represented by a linear system with an additive disturbance. From this representation, a necessary and sufficient condition relative to the contractivity of a given convex compact polyhedral set is stated. Consequently, the polyhedral set can be associated with a Lyapunov function and the local asymptotic stability of the saturated closed-loop system inside the set is guaranteed. Furthermore, it is shown how, in some particular cases, the compactness condition can be relaxed in order to ensure the asymptotic stability in unbounded polyhedra. Finally, an application of the contractivity conditions is presented in order to determine local asymptotic stability regions for the closed-loop saturated system.
\end{abstract}

Index Terms-Contractivity, control saturation, local stability, polyhedral Lypunov function.

\section{INTRODUCTION}

Consider the discrete-time system described by

$$
x(k+1)=A x(k)+B \operatorname{sat}(F x(k))
$$

where $x(k) \in \mathbb{R}^{n}, A \in \mathbb{R}^{n * n}, B \in \mathbb{R}^{m * n}, F \in \mathbb{R}^{m * n}$ and each component of the saturation term is defined, $\forall i=1, \cdots, m$, as follows:

$$
\begin{aligned}
(\operatorname{sat}(F x(k)))_{(i)} & =\operatorname{sat}\left(F_{(i)} x(k)\right) \\
& = \begin{cases}-u_{\min (i)}, & \text { if } F_{(i)} x(k)<-u_{\min (i)} \\
F_{(i)} x(k), & \text { if }-u_{\min (i)} \leq F_{(i)} x(k) \leq u_{\max (i)} \\
u_{\max (i)}, & \text { if } F_{(i)} x(k)>u_{\max (i)}\end{cases}
\end{aligned}
$$

with $u_{\min (i)}, u_{\max (i)}>0$, for $i=1, \cdots, m$. System (1) represents the closed-loop system obtained from the application of a saturating state feedback control law $u(k)=\operatorname{sat}(F x(k))$ to a linear system $x(k+1)=A x(k)+B u(k)$. In the polyhedral region

$$
\begin{aligned}
S(\mathcal{F}, \rho) & =\left\{x \in \mathbb{R}^{n} ; \mathcal{F} x \leq \rho\right\} \\
& =\left\{x \in \mathbb{R}^{n} ;-u_{\min } \leq F x \leq u_{\max }\right\}
\end{aligned}
$$

where

$$
\mathcal{F} \triangleq\left[\begin{array}{c}
F \\
-F
\end{array}\right] \text { and } \rho \triangleq\left[\begin{array}{l}
u_{\max } \\
u_{\min }
\end{array}\right]
$$

the saturation does not occur and system (1) admits the classical linear model

$$
x(k+1)=(A+B F) x(k) .
$$

Due to the saturation term, the system (1) is nonlinear. Since no particular hypothesis is made about stability of matrix $A$ and

Manuscript received April 7, 1998. Recommended by Associate Editor, H. Ozbay.

J. M. Gomes da Silva, Jr. is with the UFRGS-Department of Electrical Engineering, 90035-190 Porto Alegre-RS, Brazil (e-mail: jmgomes@iee.ufrgs.br)

S. Tarbouriech is with the L.A.A.S.-C.N.R.S., 31077 Toulouse cedex 4, France (e-mail: tarbour@laas.fr).

Publisher Item Identifier S 0018-9286(99)08605-5. about how matrix $F$ was computed, in general the global asymptotic stability of system (1) cannot be ensured [17], [15]. Thus, it is important to determine domains of initial states that are driven asymptotically to the origin under the action of the saturation control law $u(k)=\operatorname{sat}(F x(k))$. These regions are regions of local asymptotic stability, and therefore can be viewed as zones of safe operation for system (1).

In this note, we deal with the determination of such regions. In particular, we consider polyhedral regions defined as follows:

$$
S(G, w)=\left\{x \in \mathbb{R}^{n} ; G x \leq w\right\}, \quad G \in \mathbb{R}^{g * n}
$$

with $w_{(i)}>0, \forall i=1, \cdots, g$. Note that by definition $S(G, w)$ is a convex set.

The problem of determining polyhedral regions of stability contained in (or equal to) the region of linearity of system (1), i.e., $S(G, w) \subseteq S(\mathcal{F}, \rho)$ was widely studied in the literature (see, for example, [1], [3], [6], [7], [11], [18], [20], and references therein). The approaches for dealing with this problem are mainly based on the concept of positive invariance (see [5] for an interesting survey). The principle consists in guaranteeing that $S(G, w)$ is positively invariant w.r.t. the linear system (3). In this case, provided that all the eigenvalues of $(A+B F)$ are placed inside the unit circle, all the trajectories originating in $S(G, w)$ are confined in it and converge to the origin without control saturation. Note that, in general, these regions are very conservative approximations of the region of attraction of system (1).

If we are interested in determining regions of stability not contained in $S(\mathcal{F}, \rho)$, i.e., $S(G, w) \not \subset S(\mathcal{F}, \rho)$, we have to consider the nonlinear behavior of system (1). In this case, the guarantee of positive invariance of $S(G, w)$ w.r.t. system (1) (see in [9] a necessary and sufficient condition for this) is not sufficient to ensure the convergence of trajectories to the origin, even if the eigenvalues of $(A+B F)$ are strictly stable. Since the behavior of the system is nonlinear, the possible existence of limit cycles and/or parasitic equilibrium points inside $S(G, w)$ have to be considered. Hence, before concluding that the polyhedron $S(G, w)$ is also a region of asymptotic stability for system (1) it is necessary to eliminate this possibility. The verification of the existence of equilibrium points, other than the origin, inside $S(G, w)$ is trivial. Nevertheless, in general, it is not easy to verify if there exist limit cycles inside $S(G, w)$. In this case, a way to ensure the asymptotic stability inside $S(G, w)$ is to guarantee the contractivity of this set w.r.t. system (1).

Definition 1 (Contractivity): Let $\partial S(G, w)$ be the boundary of $S(G, w)$. The set $S(G, w)$ is contractive with respect to system (1) if $\forall x(k) \in \lambda_{k} \partial S(G, w), \lambda_{k} \in \mathbb{R}, 0<\lambda_{k} \leq 1$, there exists $\lambda_{k+1}, 0 \leq \lambda_{k+1}<\lambda_{k}$ such that $x(k+1) \in \lambda_{k+1} \partial S(G, w)$ for all integer $k \geq 0$.

In other words, the contractivity implies that if $x(k)$ belongs to the boundary of an internal homothetic of $S(G, w)$, then $x(k+1)$ belongs to the interior of this homothetic. Note that $x(k) \in S(G, w)$, $x(k) \neq 0$, implies that $x(k) \in \lambda \partial S(G, w)$ for some $\lambda \in \mathbb{R}$, $0<\lambda \leq 1$. Hence, if $S(G, w)$ is compact, the contractivity implies the asymptotic convergence to the origin.

To the knowledge of the authors, few papers have dealt with the determination of polyhedral regions of stability in which saturation effectively occurs as well as the study of polyhedral contractivity w.r.t. the nonlinear system (1). We can cite, for example, [16] where a method for computing polyhedral regions of stability for 
second-order continuous-time saturated systems is proposed. Hence, the aim of this note is to furnish a new method of analysis in order to guarantee the local asymptotic stability, inside polyhedral sets, of linear multivariable discrete-time systems subject to control saturation. Our approach is based on the contractivity concept.

The note is organized as follows. First, a suitable representation for the nonlinear behavior of the closed-loop system is presented. Based on this representation, a necessary and sufficient condition for guaranteeing the contractivity of a compact polyhedral set is stated. Consequently, a polyhedral Lyapunov function can be associated to the contractive set and the local asymptotic stability of the saturated closed-loop system in this set is ensured. Relaxing the compactness condition of the polyhedral set, the analysis of stability inside unbounded polyhedra is discussed. Finally, in order to determine a local asymptotic stability region for system (1), an algorithm based on linear programming is proposed for computing homothetic expansions of contractive polyhedra over the zone of nonlinear behavior of the closed-loop system.

Notations: For any vector $x \in \mathbb{R}^{n}, x \geq 0$ means that all the components of $x$, denoted $x_{(i)}$, are nonnegative. For two vectors $x, y$ of $\mathbb{R}^{n}$, the notation $x \geq y$ means that $x_{(i)}-y_{(i)} \geq 0, \forall i=1, \cdots, n$. $|x|$ is the vector composed by the absolute values of the components of $x$. For any real matrix $M$, square or not, $M_{(i)}$ denotes its $i$ th row. $M^{T}$ and $\operatorname{Ker} M$ denote, respectively, the transpose and the null space of $M$. For a polyhedral set $\mathcal{S}$, int $\mathcal{S}, \partial \mathcal{S}$, and $\partial_{i} \mathcal{S}$ denote, respectively, the interior, the boundary and the $i$ th facet of $\mathcal{S}$. Given a vector $x \in \mathbb{R}^{n}, \operatorname{diag}(x)$ denotes an $n$-order diagonal matrix generated from $x$. Finally, $1_{m} \triangleq\left[\begin{array}{lll}1 & \cdots & 1\end{array}\right]^{T} \in \mathbb{R}^{m}$ and $0_{m} \triangleq\left[\begin{array}{lll}0 & \cdots & 0\end{array}\right]^{T} \in \mathbb{R}^{m}$.

\section{SATURATEd System RePresentation}

Before studying the stability properties related to saturated systems, we define a mathematical representation for these kinds of systems. This representation consists of dividing the state space in regions called regions of saturation. Inside each region of saturation, the system (1) can be modeled as a linear system with an additive disturbance [14].

Consider a vector $\xi \in \mathbb{R}^{m}$ such that each entry $\xi_{(i)}, i=1, \cdots, m$, takes the values 1,0 , or -1 as follows.

- If $u_{(i)}=u_{\max (i)}$ then $\xi_{(i)}=1$, that is, $x$ is such that $F_{(i)} x>u_{\max (i)}$.

- If $u_{(i)}=F_{(i)} x$ then $\xi_{(i)}=0$, that is, $x$ is such that $-u_{\min (i)} \leq$ $F_{(i)} x \leq u_{\max (i)}$.

- If $u_{(i)}=-u_{\min (i)}$ then $\xi_{(i)}=-1$, that is, $x$ is such that $F_{(i)} x<-u_{\min (i)}$.

Hence, each vector $\xi$ represents a possible combination between saturated and nonsaturated control entries. There are $3^{m}$ different combinations of $\xi$. For each vector $\xi_{j}, j=1, \cdots, 3^{m}$, the state vector belongs to a specific region called region of saturation. Each region of saturation is defined by the intersection of half-spaces of type $F_{(i)} x \leq d_{(i)}$ or $-F_{(i)} x \leq d_{(i)}$, where $d_{(i)}$ can be $u_{\min (i)}$, $-u_{\min (i)}, u_{\max (i)}$, or $-u_{\max (i)}$.

Thus, by defining both $d_{j} \in \mathbb{R}^{l_{j}}$ from the entries of $u_{\max },-u_{\max }$, $u_{\min }$ and $-u_{\min }$, and $R_{j} \in \mathbb{R}^{l^{* n}}$ from the rows of $F$ and $-F$, the region of saturation associated to $\xi_{j}$ is generically defined as:

$$
S\left(R_{j}, d_{j}\right)=\left\{x \in \mathbb{R}^{n} ; R_{j} x \leq d_{j}\right\} .
$$

Notice that the region corresponding to $\xi_{j}=0_{m}$ is the polyhedron $S(\mathcal{F}, \rho)$. In the other regions, there is at least one control entry that is saturated.
Inside each region of saturation, $S\left(R_{j}, d_{j}\right)$, the motion of system (1) can be described by a linear dynamical equation given by

$$
x(k+1)=\left(A+B \operatorname{diag}\left(1_{m}-\left|\xi_{j}\right|\right) F\right) x(k)+B u\left(\xi_{j}\right)
$$

where

$$
u_{(i)}\left(\xi_{j}\right) \triangleq \begin{cases}-u_{\min (i)}, & \text { if } \xi_{j(i)}=-1 \\ 0, & \text { if } \xi_{j(i)}=0 \\ u_{\max (i)}, & \text { if } \xi_{j(i)}=1\end{cases}
$$

Generically, if $x(k) \in S\left(R_{j}, d_{j}\right)$ it follows that

$$
x(k+1)=\bar{A}_{j} x(k)+p_{j}
$$

with $\bar{A}_{j} \triangleq A+B \operatorname{diag}\left(1_{m}-\left|\xi_{j}\right|\right) F$ and $p_{j} \triangleq B u\left(\xi_{j}\right)$.

\section{Contractivity and Local Asymptotic Stability}

In this section, based on the representation defined in the previous section, we study the contractivity and the local asymptotic stability w.r.t. polyhedral sets and system (1). The idea is to divide the analysis in some polyhedral subdomains of $S(G, w)$.

Assume that $S(G, w)$ is a compact polyhedron. Let $\partial_{i} S(G, w) \triangleq\left\{x \in \mathbb{R}^{n} ; G_{(i)} x=w_{(i)}\right.$ and $G_{(l)} x \leq w_{(l)}$ if $l \neq i\}$ be the $i$ th facet of polyhedron $S(G, w)$. Define the polyhedral cone $\mathcal{K}_{i}$, generated by the facet $\partial_{i} S(G, w)$, as follows:

$$
\mathcal{K}_{i} \triangleq\left\{x \in \mathbb{R}^{n} ; \frac{G_{(l)}}{w_{(l)}} x \leq \frac{G_{(i)}}{w_{(i)}} x, \quad \forall l=1, \cdots, g, l \neq i\right\} .
$$

Consider the set $\mathcal{J} \triangleq\left\{j ; S(G, w) \cap S\left(R_{j}, d_{j}\right) \neq \emptyset\right\}$. For each $j \in \mathcal{J}$, let $\mathcal{I}_{j}$ be the set of indices $i$ such that the cone $\mathcal{K}_{i}$ has a nonempty intersection with the region $S\left(R_{j}, d_{j}\right)$, i.e., $\mathcal{I}_{j} \triangleq\{i$; $\left.\mathcal{K}_{i} \cap S\left(R_{j}, d_{j}\right) \neq \emptyset\right\}$.

Theorem 1: Consider the description of system (1) in the region of saturation $j, j \in \mathcal{J}$, given by (8). For each $i \in \mathcal{I}_{j}$ define the following linear programs for $l=1, \cdots, g$ :

$$
\operatorname{LP}_{j(l, i)}\left\{\begin{array}{c}
y_{j(l, i)}=\max _{x}\left(G_{(l)} \bar{A}_{j}-\frac{w_{(l)}}{w_{(i)}} G_{(i)}\right) x+G_{(l)} p_{j} \\
\text { subject to } \\
x \in\left(\mathcal{K}_{i} \cap S\left(R_{j}, d_{j}\right) \cap S(G, w)\right) .
\end{array}\right.
$$

Define $y_{j}=\max \left\{y_{j(l, i)} ; i \in \mathcal{I}_{j}, l=1, \cdots, g\right\}$. The polyhedron $S(G, w)$ is contractive w.r.t. system (1) if and only if the following conditions hold:

i) $y_{j}<0$ for each $j \in \mathcal{J}$ such that $S\left(R_{j}, d_{j}\right) \neq S(\mathcal{F}, \rho)$;

ii) $y_{j}=0$ for $j$ such that $S\left(R_{j}, d_{j}\right)=S(\mathcal{F}, \rho)$ and, in this case, the optimal solution to each linear program $\operatorname{LP}_{j(l, i)}$ is unique and obtained for $x=0$.

Proof:

Sufficiency: For all $x \in S(G, w), x \neq 0$, it follows that there exists $\lambda \in \mathbb{R}, 0<\lambda \leq 1$ such that $x \in \lambda \partial S(G, w)$. In other words, $x$ belongs at least to one facet of $S(G, \lambda w)$, i.e., $x \in \partial_{i} S(G, \lambda w) \triangleq\left\{x \in \mathbb{R}^{n} ; G_{(i)} x=\lambda w_{(i)}, G_{(l)} x \leq \lambda w_{(l)}\right.$, $\forall l \neq i, l=1, \cdots, g\}$ for some $i$ and it follows that $x \in \mathcal{K}_{i}$. Moreover, $x$ belongs to some region of saturation $S\left(R_{j}, d_{j}\right)$ and thus $\mathcal{K}_{i} \cap S\left(R_{j}, d_{j}\right) \neq \emptyset$ and it follows that $\partial_{i} S(G, \lambda w) \subset$ $\left(\mathcal{K}_{i} \cap S\left(R_{j}, d_{j}\right) \cap S(G, w)\right)$. Hence, since $x$ is supposed to be different from zero, if conditions i) and ii) hold it follows that $\left(G_{(l)} \bar{A}_{j}-\left(w_{(l)} / w_{(i)}\right) G_{(i)}\right) x+G_{(l)} p_{j}<0, \forall l=1, \cdots, g$, where 


$$
\begin{aligned}
& \text { if } x(k)=x: \\
& \quad G_{(l)} x(k+1)<\frac{w_{(l)}}{w_{(i)}} G_{(i)} x(k)=\lambda w_{(l)} \quad \forall l=1, \cdots, g
\end{aligned}
$$

and therefore $G x(k+1)<\lambda w$ which implies that $x(k+1) \in$ $\partial S\left(G, \lambda_{1} w\right)=\lambda_{1} \partial S(G, w)$ with $\lambda_{1} \in \mathbb{R}, 0 \leq \lambda_{1}<\lambda$. Since this reasoning can be applied $\forall x \in S(G, w), x \neq 0$, the contractivity of $S(G, w)$ is guaranteed if i) and ii) hold.

Necessity: Suppose that $S(G, w)$ is contractive and i) or ii) does not hold. Then for some some $j \in \mathcal{J}, i \in \mathcal{I}_{j}$, and $l$ there exists $x \in\left(\mathcal{K}_{i} \cap S\left(R_{j}, d_{j}\right) \cap S(G, w)\right), x \neq 0$, (i.e., $x \in \partial_{i} S(G, \lambda w)$ with $0<\lambda \leq 1)$, such that

$$
\left(G_{(l)} \bar{A}_{j}-\frac{w_{(l)}}{w_{(i)}} G_{(i)}\right) x+G_{(l)} p_{j} \geq 0 .
$$

Suppose now that $x(k)=x$ for some $k>0$. Since $x(k) \in$ $\partial_{i} S(G, \lambda w)$, it follows that $G_{(i)} x(k)=\lambda w_{(i)}$ and we obtain $G_{(l)}\left(\bar{A}_{j} x(k)+p_{j}\right)-\lambda w_{(l)} \geq 0$, i.e., $G_{(l)} x(k+1) \geq \lambda w_{(l)}$. Therefore it follows that $x(k+1) \in \partial S\left(G, \lambda_{1} w\right), \lambda_{1} \geq \lambda$ which contradicts the hypothesis that $S(G, w)$ is contractive and thus the necessity of conditions i) and ii) is proven.

The principle of Theorem 1 is to analyze the trajectories behavior of system (1) in some subdomains of $S(G, w)$. These subdomains are delimited by a polyhedral cone $\mathcal{K}_{i}$ and a region of saturation $S\left(R_{j}, d_{j}\right)$. For any $x(k)$ belonging to $S(G, w)$, it follows, that $x(k)$ belongs both to one of these subdomains and an $i$ th facet of an internal homothetic of $S(G, w)(\lambda S(G, w), 0<\lambda \leq 1)$. In order to prove the contractivity of the domain, we must guarantee that $x(k+1)$ verifies $G x(k+1)<\lambda w$. Since, in each subdomains $x(k+1)$ is described from (8), this condition is equivalent to have $\left(G_{(l)} \bar{A}_{j}-\left(w_{(l)} / w_{(i)}\right) G_{(i)}\right) x+G_{(l)} p_{j}<0, \forall l=1, \cdots, g$. Roughly speaking, this condition is tested by means of linear programs (10) for all possible vector $x(k)$ belonging to $S(G, w)$. Thus, Theorem 1 provides a condition that guarantees the contractivity of all the trajectories originating in $S(G, w)$. Hence, it is ensured that there is neither limit cycle nor parasitic equilibrium points inside $S(G, w)$. Since $S(G, w)$ is a convex compact polyhedron it is possible to associate to it a Lyapunov polyhedral function [3], [4], [10], [18]. This function is strictly decreasing along all the trajectories of system (1) originating in $S(G, w)$. Hence, we can state the following corollary.

Corollary 1: If conditions of Theorem 1 hold, then we have the following.

i) System (1) is locally asymptotically stable in $S(G, w)$.

ii) The polyhedral function

$$
\mathcal{V}(x(k))=\max _{i}\left\{\frac{G_{(i)} x(k)}{w_{(i)}}\right\}
$$

is a strictly decreasing Lyapunov function for system (1).

Proof: i) It follows directly from the fact that $S(G, w)$ is compact, contains the origin and is contractive. ii) Since $S(G, w)$ is compact and contains the origin, it follows that $\mathcal{V}(x(k))>0$, $\forall x(k) \neq 0$ and $\mathcal{V}(x(k))=0$ if and only if $x(k)=0$. Since $S(G, w)$ is contractive, we have $\mathcal{V}(x(k+1))-\mathcal{V}(x(k))<0$, $\forall x(k) \in S(G, w), x(k) \neq 0, \forall k \geq 0$. Thus $\mathcal{V}(x(k))$ is a strictly decreasing Lyapunov function for system (1).

\section{UnBounded POLYHEdRA}

From Theorem 1, we can conclude stability in $S(G, w)$ only if it is a compact set. However, in some cases, the condition of compactness can be relaxed as we will show in this section. For this, consider the following assumption.
Assumption 1: Consider that $S(G, w)$ is unbounded and there exists $0<\beta, \beta \in \mathbb{R}$, such that $S(G, \beta w) \subseteq S(\mathcal{F}, \rho)$. Suppose also that $\operatorname{Ker} G$ is an $A$-invariant subspace.

This assumption implies that $\mathcal{K}$ er $G \subseteq \mathcal{K}$ er $F$. In this case, a necessary condition to the contractivity of $S(G, w)$ w.r.t. the system (1) is that $\operatorname{Ker} G$ is an $A$-invariant subspace [7].

Consider $Q=\left[\begin{array}{ll}Q_{o} & Q_{r}\end{array}\right], Q \in \mathbb{R}^{n * n}$, where $Q_{o} \in \mathbb{R}^{n *(n-g)}$ is a basis for the subspace $\mathcal{S}_{o} \triangleq \mathcal{K} \operatorname{er} G$ and $Q_{r}$ is a basis for the complementary subspace of $\mathcal{S}_{o}$ denoted by $\mathcal{S}_{r}$. Define the change of basis: $x=\left[\begin{array}{ll}Q_{o} & Q_{r}\end{array}\right]\left[\begin{array}{ll}z_{o}^{T} & z_{r}^{T}\end{array}\right]^{T}$.

Since $\operatorname{Ker} G$ is supposed to be $A$-invariant and $\operatorname{Ker} G \subseteq \mathcal{K}$ er $F$ it follows that $F Q_{o}=0$ and system (1) can be rewritten in the new basis as

$$
\begin{aligned}
& z_{o}(k+1)=R_{o} z_{o}(k)+R_{2} z_{r}(k)+B_{o} \operatorname{sat}\left(F_{r} z_{r}(k)\right) \\
& z_{r}(k+1)=R_{r} z_{r}(k)+B_{r} \operatorname{sat}\left(F_{r} z_{r}(k)\right) .
\end{aligned}
$$

The projection of the polyhedron $S(G, w)$ in $\mathcal{S}_{r}$ along $\mathcal{S}_{o}$ is given by

$$
S\left(G_{r}, w\right) \triangleq\left\{z_{r} \in \mathbb{R}^{g} ; G_{r} z_{r} \leq w\right\}
$$

with $G_{r}=G Q_{r}$.

Property 1: The unbounded polyhedron $S(G, w)$, under Assumption 1 , is a contractive set for system (1) if and only if $S\left(G_{r}, w\right)$ is a contractive set for system (14).

Proof: Recalling that $G x=G\left[\begin{array}{ll}Q_{o} & Q_{r}\end{array}\right]\left[\begin{array}{ll}z_{o}^{T} & z_{r}^{T}\end{array}\right]^{T}=$ $\left[\begin{array}{ll}0 & G_{r}\end{array}\right]\left[\begin{array}{ll}z_{o}^{T} & z_{r}^{T}\end{array}\right]^{T}=G_{r} z_{r}$ then the proof directly follows.

Proposition 1: Consider that $S(G, w)$ is an unbounded polyhedron satisfying Assumption 1. If $S\left(G_{r}, w\right)$ is contractive for system (14) and all the eigenvalues of matrix $R_{o}$ are located inside the unit disk of the complex plane, then $S(G, w)$ is a domain of local asymptotic stability for system (1).

Proof: Since $S\left(G_{r}, w\right)$ is a compact set, if $S\left(G_{r}, w\right)$ is a contractive set, it is also a domain of local asymptotic stability for the reduced order system (14). It follows that $z_{r}(k) \rightarrow 0$ as $k \rightarrow \infty$, $\forall z_{r}(0) \in S\left(G_{r}, w\right)$. Since all the eigenvalues of $R_{o}$ are located inside the unit disk of the complex plane, from Theorem 1 in [15] we can conclude that $z_{o}(k) \rightarrow 0$ as $k \rightarrow \infty, \forall z_{r}(0) \in S\left(G_{r}, w\right)$, $\forall z_{o}(0) \in \mathbb{R}^{n-g}$. Since $z_{o}(k) \in \mathcal{K}$ er $G, \forall k \geq 0$, it follows that $\forall x(0) \in S(G, w)$, the corresponding trajectory converges asymptotically to the origin.

Thus, under Assumption 1, we can conclude that the contractivity and the local asymptotic stability inside an unbounded polyhedron $S(G, w)$ can be verified by considering a reduced order system with saturating controls and the projection of $S(G, w)$ in the complementary subspace of $\mathcal{K}$ er $G$. Since $S\left(G_{r}, w\right)$ is compact, the verification is accomplished by applying Theorem 1 .

The use of Assumption 1 means that we consider a particular case. Note that when Assumption 1 is satisfied and all the eigenvalues of matrix $R_{o}$ are located inside the unit disk of the complex plane, the basin of attraction of the origin for system (1) is unbounded in the directions of $\mathcal{K} \operatorname{er} G$. It should be pointed out that, without this assumption, we cannot directly apply the results of Section III. In particular, when $\mathcal{K} \operatorname{er} G \nsubseteq \mathcal{K}$ er $F$, system (13)-(14) is not equivalent to system (1).

\section{Determination of LOCAL AsYMPtOtic Stability Regions}

In this section, we show how to use the contractivity condition stated in Theorem 1 in order to determine compact polyhedral regions of local asymptotic stability for the system (1). 


\section{Determination of Local Asymptotic Stability Regions}

In this section, we show how to use the contractivity condition stated in Theorem 1 in order to determine compact polyhedral regions of local asymptotic stability for the system (1).

\section{A. Numerical Procedure}

The problem of determining contractive polyhedral sets contained in the region of linear behavior (2) of the saturated system has been addressed in many papers. For example, by using the method described in [4] and [8] it is possible to compute the maximal invariant and contractive set contained in $S(\mathcal{F}, \rho)$ for the linear model (3). Furthermore, there exist techniques based on eigenstructure assignment (see, for example, [11] for an interesting survey) and linear programming [20] for computing a matrix $F$ which makes contractive a given set $S(G, w)$ and $S(G, w) \subseteq S(\mathcal{F}, \rho){ }^{1}$

Hence, if we assume that a compact polyhedron $S(G, w)$, contractive with respect to the linear model (3), was computed from one of the techniques found in the literature, we can apply Theorem 1 for calculating the maximum coefficient of homothesis, $\delta_{\max }$, for which $S\left(G, \delta_{\max } w\right)$ preserves the property of contractivity w.r.t. the saturated system (1). The algorithm is based on iterative computations and linear programming schemes.

Algorithm:

- Step 0: Initialize $\delta=\delta_{0}$. Choose a computational accuracy.

- Step 1: Determine $\mathcal{J}$ w.r.t. $S(G, \delta w)$. For each $j \in \mathcal{J}$ and $i \in \mathcal{I}_{j}$, solve the following linear programs, $l=1, \cdots, g$ :

$$
\left\{\begin{array}{c}
y_{j(l, i)}=\max _{x}\left(G_{(l)} \bar{A}_{j}-\frac{w_{(l)}}{w_{(i)}} G_{(i)}\right) x+G_{(l)} p_{j} \\
\text { subject to } \\
{\left[\begin{array}{c}
R_{j} \\
G
\end{array}\right] x \leq\left[\begin{array}{c}
d_{j} \\
\delta w
\end{array}\right]} \\
\left(\frac{G_{(l)}}{w_{(l)}}-\frac{G_{(i)}}{w_{(i)}}\right) x \leq 0 \quad \forall l \neq i .
\end{array}\right.
$$

- Step 2: For each $j \in \mathcal{J}$ compute $y_{j}=\max \left\{y_{j(l, i)} ; i \in \mathcal{I}_{j}, l=\right.$ $1, \cdots, g\}$. If conditions i) and ii) of Theorem 1 hold, go to Step 4 otherwise go to Step 3.

- Step 3: Decrease $\delta$ and return to step 1.

- Step 4: If the difference between the $\delta$ of this iteration and the last iteration is greater than the chosen accuracy, increase $\delta$. Otherwise stop: $\delta_{\max }=\delta$.

When $S(G, w)$ is compact, the maximal homothetic set to $S(G, w)$, for which the asymptotic stability is guaranteed by Theorem 1 , can be considered as a conservative approximation of the region of attraction of the origin [17] for system (1). This conservativeness is inherent to the use of Lyapunov functions that lead, by definition, to convex domains [12]. Actually, in general the basin of attraction of the origin is nonconvex and not analytically determinable. In order to overcome this problem, we can consider, for example, the union of different polyhedra $\bigcup_{i=1}^{s} S\left(G_{i}, \delta_{\max i} w_{i}\right)$.

In this case, the final domain of stability may be enlarged and may be nonconvex. Moreover, this algorithm can be used in a complementary way to the one proposed in [19] in order to obtain nonhomothetic expansions of a contractive set w.r.t. to system (1).

In particular, if we have determined an initial contractive unbounded polyhedron $S(G, w)$ contained in $S(\mathcal{F}, \rho)$ (see, for instance, [7] and references therein), we can use the result of Propo-

${ }^{1}$ In these cases, matrices $F, G$, and vector $w$ satisfy in particular the properties $G(A+B F)=H G$ and $H w<w$, where $H$ is a nonnegative matrix [1], [3], [7]. They also satisfy $N G=\mathcal{F}$ and $N w \leq \rho$ where $N$ is a nonnegative matrix [11]. Note that $F$ is such that matrix $A+B F$ is asymptotically stable and the origin belongs to $S(G, w)$. sition 1 in order to generate an unbounded region of asymptotic stability for system (1) not included in $S(\mathcal{F}, \rho)$. In this case it suffices to apply the above algorithm considering the reduced order system (14) and the polyhedron $S\left(G_{r}, \delta w\right)$. The obtained $\delta_{\max }$ is such that $S\left(G, \delta_{\max } w\right)$ is an unbounded region of asymptotic stability and nonlinear behavior for system (1).

\section{B. Numerical Example}

Consider system (1) described by the following data:

$$
\begin{array}{rlrl}
A & =\left[\begin{array}{cc}
1.2 & 0 \\
0.4 & 0.5
\end{array}\right] ; & B & =\left[\begin{array}{cc}
1 & 0 \\
0 & 0.5
\end{array}\right] \\
F & =\left[\begin{array}{cc}
-0.95 & 0 \\
2.2 & 0.3
\end{array}\right] ; & u_{\max }=u_{\min }=\left[\begin{array}{l}
2 \\
4
\end{array}\right] .
\end{array}
$$

by

Consider a contractive polyhedral set $S(G, w) \subset S(\mathcal{F}, \rho)$ given

$$
G=\left[\begin{array}{rr}
1 & 0 \\
0 & 1 \\
-1 & 0 \\
0 & -1
\end{array}\right] ; \quad w=\left[\begin{array}{c}
1 \\
4.5 \\
1 \\
4.5
\end{array}\right]
$$

Matrix $F$ and control constraints define nine regions of saturation. Since the polyhedra $S(G, w)$ and $S(\mathcal{F}, \rho)$ are symmetric, we can analyze only five of these regions:

Region $1\left(\xi_{1}=\left[\begin{array}{ll}0 & 0\end{array}\right]^{T} \Leftrightarrow\right.$ Reg. Linearity):

$$
\begin{array}{lll}
\bar{A}_{1}=A+B F=\left[\begin{array}{cc}
0.25 & 0 \\
1.5 & 0.65
\end{array}\right] ; & R_{1}=\left[\begin{array}{c}
F \\
-F
\end{array}\right] \\
p_{1}=\left[\begin{array}{l}
0 \\
0
\end{array}\right] ; & d_{1}=\left[\begin{array}{l}
2 \\
4 \\
2 \\
4
\end{array}\right] .
\end{array}
$$

Region $2\left(\xi_{2}=\left[\begin{array}{ll}1 & 1\end{array}\right]^{T}\right)$ :

$$
\begin{aligned}
\bar{A}_{2} & =A ; & R_{2} & =\left[\begin{array}{cc}
0.95 & 0 \\
-2.2 & -0.3
\end{array}\right] \\
p_{2} & =\left[\begin{array}{l}
2 \\
2
\end{array}\right] ; & d_{2} & =\left[\begin{array}{l}
-2 \\
-4
\end{array}\right] .
\end{aligned}
$$

Region $3\left(\xi_{3}=\left[\begin{array}{ll}0 & 1\end{array}\right]^{T}\right)$ :

$$
\begin{array}{rlrl}
\bar{A}_{3} & =\left[\begin{array}{cc}
0.25 & 0 \\
0.4 & 0.5
\end{array}\right] ; & R_{3}=\left[\begin{array}{rc}
0.95 & 0 \\
-0.95 & 0 \\
-2.2 & -0.3
\end{array}\right] \\
p_{3}=\left[\begin{array}{l}
0 \\
2
\end{array}\right] ; & d_{3}=\left[\begin{array}{r}
2 \\
2 \\
-4
\end{array}\right] .
\end{array}
$$

Region $4\left(\xi_{4}=\left[\begin{array}{ll}-1 & 1\end{array}\right]^{T}\right)$ :

$$
\begin{aligned}
\bar{A}_{4} & =A ; & R_{4} & =\left[\begin{array}{cc}
-0.95 & 0 \\
-2.2 & -0.3
\end{array}\right] \\
p_{4} & =\left[\begin{array}{r}
-2 \\
2
\end{array}\right] ; & d_{4}=\left[\begin{array}{l}
-2 \\
-4
\end{array}\right] . &
\end{aligned}
$$

Region $5\left(\xi_{5}=\left[\begin{array}{ll}-1 & 0\end{array}\right]^{T}\right)$ :

$$
\begin{array}{rlr}
\bar{A}_{5}=\left[\begin{array}{cc}
1.2 & 0 \\
1.5 & 0.65
\end{array}\right] ; & R_{5}=\left[\begin{array}{rc}
-0.95 & 0 \\
2.2 & 0.3 \\
-2.2 & -0.3
\end{array}\right] \\
p_{5}=\left[\begin{array}{r}
-2 \\
0
\end{array}\right] ; & d_{5}=\left[\begin{array}{r}
-2 \\
4 \\
4
\end{array}\right] .
\end{array}
$$




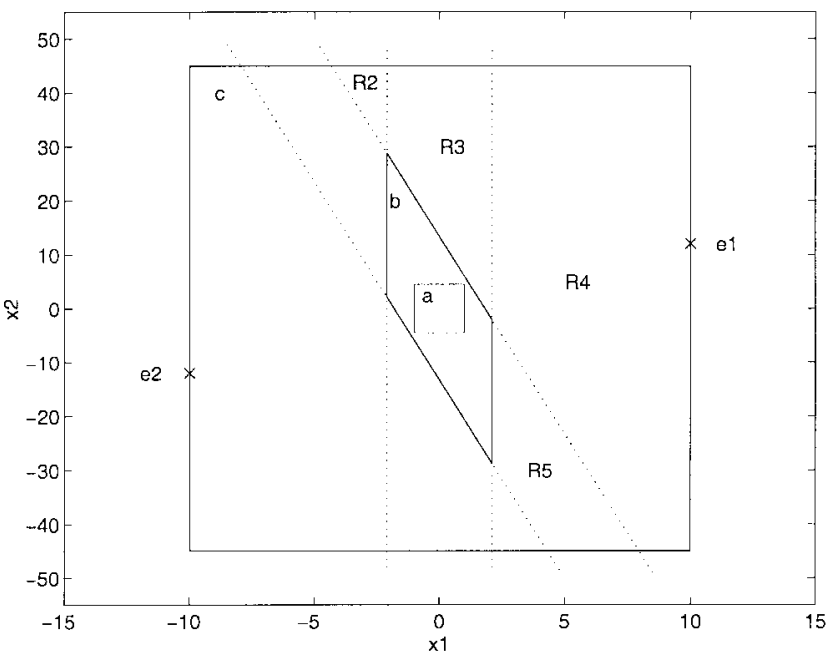

Fig. 1. (a) $S(G, w)$, (b) $S(\mathcal{F}, \rho)$, and (c) $S\left(G, \delta_{\max } w\right)$.

By applying the algorithm described in Section V-A, one obtains $\delta_{\max }=9.99$ (accuracy $\left.=0.01\right) . S\left(G, \delta_{\max } w\right)$ represents the maximal homothetic set of $S(G, w)$ which is contractive w.r.t. the closed-loop saturated system. Since it is a compact set, from Corollary 1 , it is a domain of asymptotic stability and a safe operation for system (1).

The maximal homothetic positively invariant set is obtained for $\delta=10$ (see [9]). In this case, there exist some trajectories that remain on the boundary of the domain. This fact is justified by the existence of parasitic equilibrium points on the boundary of the set $\left(e_{1}=\left[\begin{array}{ll}10 & 12\end{array}\right]^{T}, e_{2}=\left[\begin{array}{ll}-10 & -12\end{array}\right]^{T}\right)$. For $\delta>10$ one gets unstable trajectories originating in the domain.

It is worth to notice that the maximal homothetic set to $S(G, w)$ contained in the region of linearity $S(\mathcal{F}, \rho)$ is obtained for $\delta=$ 1.1268. Fig. 1 depicts $S(G, w), S(\mathcal{F}, \rho), S\left(G, \delta_{\max } w\right)$, the parasitic equilibrium points $\left(e_{1}\right.$ and $\left.e_{2}\right)$ of the saturated system and the regions of saturation $\left(R_{j}\right.$ 's).

\section{CONCLUSION}

In this note, we considered the analysis of local stability inside polyhedral regions for discrete-time systems with saturating controls. This analysis was based on the property of contractivity of such polyhedral sets. In this sense, a necessary and sufficient condition was stated in order to guarantee the contractivity of a compact polyhedral set having nonempty intersection with the nonlinear behavior region of the saturated system. In this case, there exists a Lyapunov polyhedral function, strictly decreasing, for all the states belonging to the considered polyhedral set. Consequently, the local asymptotic stability of the saturated system is ensured. An algorithm based on linear programming was proposed to compute polyhedral regions of stability and nonlinear behavior (i.e., the controls effectively saturate in these regions) for the closed-loop system. Since the exact determination of the region of attraction of the origin is, in general, not possible for systems with saturating controls, the use of the proposed algorithm can be seen as an interesting way to compute approximations of this region.

The results presented in this note considered the case of state feedback. Nevertheless, their application to the case of output feedback (static or dynamic) is straightforward. In this case, we have to redefine the region of linearity and the regions of saturation in function of the matrices that define the considered feedback. The proposed approach should also allow to treat the problem of systems with saturating controls and with both additive and input disturbances. This will be addressed by the authors in a forthcoming publication.

\section{REFERENCES}

[1] A. Benzaouia and C. Burgat, "Regulator problem for linear discrete-time systems with nonsymmetrical constrained control," Int. J. Contr., vol. 48 , no. 6 , pp. $2441-2451,1988$.

[2] D. S. Bernstein and A. N. Michel, "A chronological bibliography on saturating actuators," Int. J. Robust Nonlinear Contr., vol. 5, pp. 375-380, 1995 .

[3] G. Bitsoris, "On the positive invariance of polyhedral sets for discretetime systems," Syst. Contr. Lett., vol. 11, pp. 243-248, 1988.

[4] F. Blanchini, "Ultimate boundedness control for discrete-time uncertain system via set-induced Lyapunov functions," IEEE Trans. Automat. Contr., vol. 38, pp. 428-433, 1994.

[5] _ , "Set invariance in control-A survey," personal communication, Feb. 1998.

[6] C. Burgat and S. Tarbouriech, "Stability and control of saturated linear systems," in Nonlinear Systems: Stability and Stabilization, A. J. Fossard and D. Normand-Cyrot, Eds. London: Chapman \& Hall, 1996, vol. 2, ch. 4.

[7] E. B. Castelan, J. M. Gomez da Silva, Jr., and J. E. R. Cury, "A reduced order framework applied to linear systems with constrained controls," IEEE Trans. Automat. Contr., vol. 41, pp. 249-255, 1996.

[8] E. G. Gilbert and K. T. Tan, "Linear systems with state and control constraints: The theory and application of maximal output sets," IEEE Trans. Automat. Contr., vol. 36, pp. 1008-1019, 1991.

[9] J. M. Gomez da Silva, Jr., and S. Tarbouriech, "Polyhedral regions of local stability for linear discrete-time systems with saturating controls," in Proc. 36th IEEE CDC, San Diego, CA, Dec. 1997, vol. 1, pp. 925-930.

[10] H. Kiendl, J. Adamy, and P. Stelzner, "Vector norms as Lyapunov functions for linear systems," IEEE Trans. Automat. Contr., vol. 37, pp. 839-842, 1992

[11] J. C. Hennet, "Discrete time constrained linear systems," in Control and Dynamic Systems. New York: Academic, 1995, vol. 71, pp. 157-213.

[12] H. J. Khalil, Nonlinear Systems. New York: Macmillan, 1992.

[13] D. G. Luenberger, Linear and Nonlinear Programming, 2nd ed. Reading, MA: Addison-Wesley, 1984.

[14] T. C. T. Rocha, "Positively invariant domains of linear systems with constraints on the control variables," M.Sc. thesis, LCMI/PGEEL, UFSC, Florianópolis, Brazil, July 1994 (in Portuguese).

[15] Y. Yang, J. Sussmann, and E. Sontag, "Stabilization of linear systems with bounded controls," in Proc. NOLCOS, Bordeaux, France, June 1992, pp. 15-20.

[16] B. G. Romanchuck, "Computing regions of attraction with polytopes: Planar case," Automatica, vol. 32, no. 12, pp. 1727-1732, 1996.

[17] R. Suarez, J. Alvarez-Ramirez, and J. Alvarez, "Linear systems with single saturated input: Stability analysis," in Proc. 30th IEEE-CDC, Brighton, England, Dec. 1991, pp. 223-228.

[18] M. Sznaier, "A set induced norm approach to the robust control of constrained systems," SIAM J. Contr. Optim., vol. 31, no. 3, pp. 733-746, 1993.

[19] S. Tarbouriech and J. M. Gomez da Silva, Jr., "Admissible polyhedra for discrete-time linear systems with saturating controls," in Proc. 1997 Amer. Contr. Conf., Albuquerque, NM, June 1997, vol. 6, pp. 3915-3919.

[20] M. Vassilaki, J. C. Hennet, and G. Bitsoris, "Feedback control of linear discrete-time systems under state and control constraints," Int. J. Contr. vol. 47 , pp. 1727-1735, 1988. 